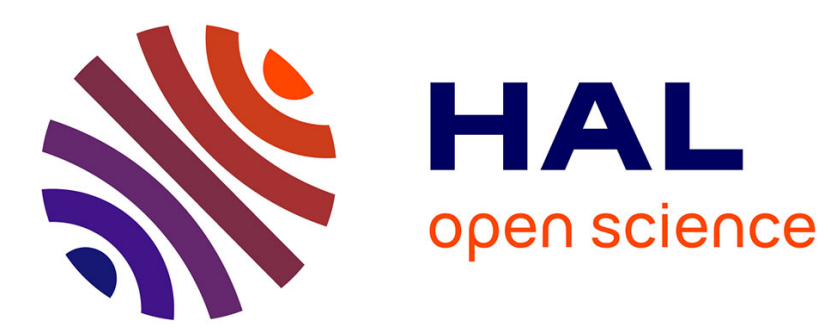

\title{
The effect of a lifted flame on the stability of round fuel jets
}

Joseph W. Nichols, Peter Schmid

\section{To cite this version:}

Joseph W. Nichols, Peter Schmid. The effect of a lifted flame on the stability of round fuel jets. Journal of Fluid Mechanics, 2008, 609 (august), pp.275-284. 10.1017/s0022112008002528 . hal-01022810

\section{HAL Id: hal-01022810}

https: / hal-polytechnique.archives-ouvertes.fr/hal-01022810

Submitted on 20 Jul 2014

HAL is a multi-disciplinary open access archive for the deposit and dissemination of scientific research documents, whether they are published or not. The documents may come from teaching and research institutions in France or abroad, or from public or private research centers.
L'archive ouverte pluridisciplinaire HAL, est destinée au dépôt et à la diffusion de documents scientifiques de niveau recherche, publiés ou non, émanant des établissements d'enseignement et de recherche français ou étrangers, des laboratoires publics ou privés. 


\title{
The effect of a lifted flame on the stability of round fuel jets
}

\author{
JOSEPH W. NICHOLS AND PETER J. SCHMID \\ Laboratoire d'Hydrodynamique (LadHyX), CNRS-École Polytechnique, \\ 91128 Palaiseau, France
}

(Received 26 March 2008 and in revised form 23 May 2008)

The stability and dynamics of an axisymmetric lifted flame are studied by means of direct numerical simulation (DNS) and linear stability analysis of the reacting low-Mach-number equations. For light fuels (such as non-premixed methane/air flames), the non-reacting premixing zone upstream of the lifted flame base contains a pocket of absolute instability supporting self-sustaining oscillations, causing flame flicker even in the absence of gravity. The liftoff heights of the unsteady flames are lower than their steady counterparts (obtained by the method of selective frequency damping (SFD)), owing to premixed flame propagation during a portion of each cycle. From local stability analysis, the lifted flame is found to have a significant stabilizing influence at and just upstream of the flame base, which can truncate the pocket of absolute instability. For sufficiently low liftoff heights, the truncated pocket of absolute instability can no longer support self-sustaining oscillations, and the flow is rendered globally stable.

\section{Introduction}

Lifted flames are important to a number of industrial applications such as efficient gas turbine design, flare burner development, and even diesel engines (Tap \& Veynante 2005). A lifted flame forms on a non-premixed fuel jet when the flow rate of the fuel is large enough that the heat flux caused by the local fluid strain rate surpasses the heat source from combustion in the immediate vicinity of the nozzle. In this case, the flame cannot be sustained at the initial fuel/air interface anchored to the nozzle rim, but will instead lift off the nozzle, and stabilize at a distance downstream. Since the flame does not touch the nozzle in a lifted-flame configuration, fuel nozzle corrosion can be minimized which is an important issue in boilers designed for large-scale energy production.

Laboratory experiments and numerical simulations have shown that laminar lifted flames have a triple flame structure at the stabilization location (Ghosal \& Vervisch 2001; Linán et al. 2005; Won et al. 2005). Joedicke, Peters \& Mansour (2005) have observed this phenomenon in laboratory low-velocity turbulent lifted flames as well. Therefore, understanding the interaction of triple flames with flow structures is key to understanding the physics in the stabilization region of turbulent lifted flames.

As is often the case in combustion applications, the unburnt fuel density $\rho_{j}$ may be different than that of the unburnt oxidizer $\rho_{0}$. For a small enough density ratio $S=\rho_{j} / \rho_{0}$, it has been shown that a variable-density jet contains a pocket of absolute instability from the nozzle exit to a distance downstream (Monkewitz \& Sohn 1988; Lesshafft et al. 2006; Nichols, Schmid \& Riley 2007). The global instability of the flow then can be understood as follows: the pocket of absolute instability serves as 


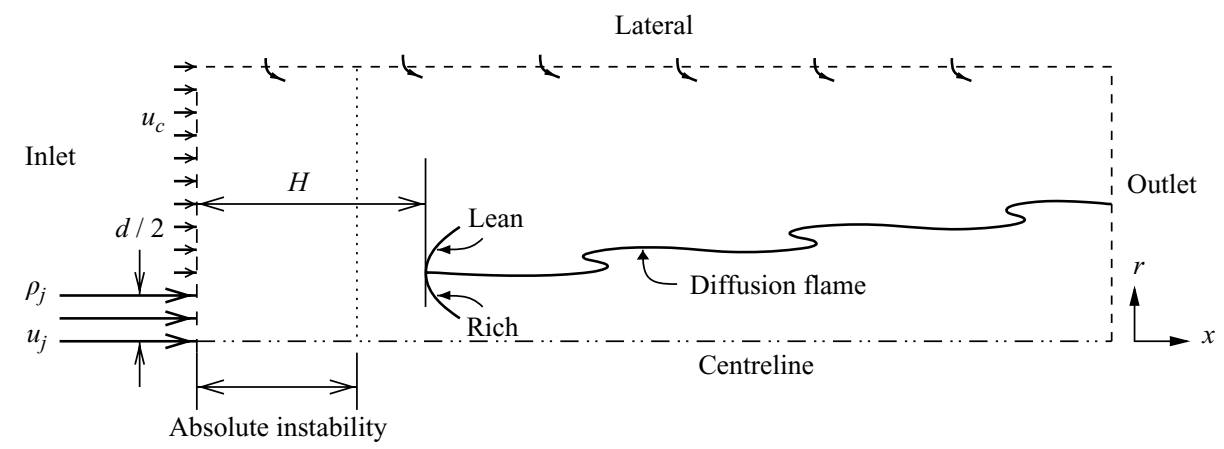

FIGURE 1. Schematic of an axisymmetric lifted flame. A fuel jet with a small coflow of air enters at the left. A lifted flame forms at a distance $H$ from the inlet, which is shown here to be downstream of the pocket of absolute instability (inlet to dotted line).

a 'wavemaker', producing oscillations which then convect and amplify downstream (Chomaz 2005). In this paper, we explore this idea by examining the effect of gradually lowering the base of a lifted flame into the pocket of absolute instability. This is accomplished by means of direct numerical simulations (DNS) in which we can easily vary flow parameters such as the Damköhler number, $D a$. We hypothesize (and confirm) that below a critical liftoff height, the flame renders the spatial extent of the pocket of absolute instability sufficiently small that it can no longer support an unstable global mode, and thus stabilizes the entire flow.

\section{Mathematical model and numerical method}

\subsection{Flow geometry}

Figure 1 shows a schematic of the flow configuration. An axisymmetric jet of pure fuel with velocity $u_{j}$ and density $\rho_{j}$ enters through a circular nozzle of diameter $d$ at the left. At the inlet, the jet is surrounded by a small coflow of oxidizer with velocity $u_{c}=0.01 u_{j}$. A version of Michalke's profile number two (Michalke 1984) is used as a functional form for both the velocity $u_{x}(0, r)$ and fuel-to-air mixture fraction $Z(0, r)$ at the inlet:

$$
\begin{gathered}
f(r)=\frac{1}{2}\left(1+\tanh \left(\frac{1}{4} \frac{d}{2 \theta}\left(\frac{d}{2 r}-\frac{2 r}{d}\right)\right)\right), \\
u_{x}(0, r)=\left(u_{j}-u_{c}\right) f(r)+u_{c}, \quad Z(0, r)=f(r),
\end{gathered}
$$

where the ratio of the jet radius $d / 2$ to the momentum thickness $\theta$ was taken to be $d /(2 \theta)=12.5$. In addition to the coflow, fresh oxidizer may also enter the domain by entrainment through the open lateral boundaries.

Once ignited, a flame forms on the jet. The largest reaction rates occur in regions where the local stoichiometry of the fuel/air mixture is correct for complete combustion. Thus, the resulting flame follows a surface of constant stoichiometry within the domain. This surface begins at the nozzle lip, where the fuel first meets the air, so that low-flow-rate flames anchor themselves to the nozzle. As previously mentioned, however, for sufficiently large flow rates $u_{j}$ the flame lifts off the nozzle, and stabilizes a distance $H$ downstream, as shown in figure 1 . We define $H$ by the minimum axial location $x$ such that the reduced temperature $T>0.5$ for any $r$. Various theories have been proposed to predict the liftoff height, including the premixed flame speed (Vanquickenborne \& van Tiggelen 1966) and flamelet quenching (Peters \& Williams 1983). The partial premixing of the fuel in the zone upstream of the flame 
front produces a characteristic triple flame structure (Ghosal \& Vervisch 2000) at the base of the lifted flame. The triple flame structure consists of rich and lean premixed branches that serve to anchor a diffusion flame, which extends for a large distance downstream, following the stoichiometric surface.

\subsection{Governing equations and methodology}

The flow is described by the axisymmetric reacting low-Mach-number equations (see McMurtry 1987). The formulation of the low-Mach-number equations used in this paper is the same as in Nichols et al. (2007), except that the energy equation is modified to include a source term Daw, an equation governing the transport of a conserved scalar (mixture fraction) is added, and the equation of state is modified to include a dependence on both reduced temperature $T$ and mixture fraction $Z$ :

$$
\begin{gathered}
\rho \frac{\mathrm{D} T}{\mathrm{D} t}=\frac{S}{\operatorname{RePr}} \nabla^{2} T+\text { Daw }, \quad \rho \frac{\mathrm{D} Z}{\mathrm{D} t}=\frac{S}{\operatorname{ReSc}} \nabla^{2} Z, \\
1=\rho\left[\frac{1-S}{S} Z+1\right]\left[\frac{\alpha}{1-\alpha} T+1\right] .
\end{gathered}
$$

The added source term Daw represents heat released by reaction. In the current study, the chemistry is modelled by a simple one-step reversible reaction of the form

$$
Y_{F}+r^{*} Y_{O} \rightleftharpoons\left(1+r^{*}\right) Y_{P},
$$

where $Y_{F}, Y_{O}$, and $Y_{P}$ are the mass fractions of fuel, oxidizer, and product, respectively. Here, $r^{*}$ defines a stoichiometric ratio of oxidizer to fuel necessary for complete combustion. In the simulations that follow, $r^{*}=2$ giving a stoichiometric mixture fraction $Z_{s t}=0.333$, which occurs near the jet shear layer so that the resulting flame will interact strongly with the instability of the flow. Also, we assume a unit Lewis number with $\operatorname{Pr}=S c=0.7$, a value commonly used for air. In this case, the evolution equations for product mass fraction $Y_{P}$ and reduced temperature $T$ are identical so $Y_{P}=T$. Since $Y_{F}+Y_{O}+Y_{P}=1$, the fluid composition and temperature can be completely described by two independent variables: the mixture fraction, $Z$, and the progress variable, $T$ (Sripakagorn et al. 2004). An Arrhenius form for the reaction rate is used:

$$
\dot{w}=\rho^{2}\left(\left(Z-\frac{T}{r^{*}+1}\right)\left(1-Z-\frac{r^{*} T}{r^{*}+1}\right)-\kappa T^{2}\right) \exp \left[\frac{-\beta(1-T)}{1-\alpha(1-T)}\right],
$$

where $\kappa=0.01$ is an equilibrium constant representing reversible chemistry, $\alpha=\left(T_{f}-\right.$ $\left.T_{0}\right) / T_{f}=0.833$ is the heat release parameter and $\beta=\alpha T_{a} / T_{f}=3.0$ is the Zeldovich number. Here, $T_{0}, T_{f}$ and $T_{a}$ denote the ambient, the adiabatic flame and the activation temperatures, respectively. While $\alpha=0.833$ is typical of real flames, $\beta$ in a real flame is measured to be around 8 (Poinsot \& Veynante 2005). Our choice of $\beta=3$, which was the maximum value allowed by the numerical resolution, means that the simulated flames are thicker than those in nature. They were found to be adequately thin, however, especially when compared to the axial wavelength of the hydrodynamic instability, to sufficiently model the essential physics of the flame-flow interaction.

In equations (2.3), $S$ specifies the density ratio of unburnt fuel to air. In the simulations, $S=0.143$, which is well within the regime of absolute instability for the jet Reynolds number $R e=1000$ considered (see Monkewitz \& Sohn 1988; Nichols et al. 2007), even in the presence of a $1 \%$ coflow. Note that the maximum possible density ratio arises from a combination of mixture and heating effects as $1 /(S(1-\alpha))=42$, but since the maximum temperature does not usually coincide with a region of pure fuel, the maximum density ratio measured from the simulations was $\approx 18.5$. 


\begin{tabular}{lccc}
\multicolumn{1}{c}{$D a$} & $S t$ & $H_{s}$ & $H_{m}$ \\
0 & 0.212 & - & - \\
$3.0 \times 10^{5}$ & 0.238 & $>10.0$ & 1.231 \\
$4.0 \times 10^{5}$ & 0.267 & 4.878 & 1.026 \\
$5.0 \times 10^{5}$ & 0.284 & 1.913 & 0.949 \\
$6.0 \times 10^{5}$ & 0.284 & 0.865 & 0.865 \\
$7.0 \times 10^{5}$ & stable & 0.557 & 0.557
\end{tabular}

TABle 1. Direct numerical simulations.

The non-dimensional Damköhler number is defined as

$$
D a=\left(1+r^{*}\right) \frac{\Delta h}{c_{p}\left(T_{f}-T_{0}\right)} \frac{A d}{u_{j}},
$$

where $\Delta h, c_{p}$ and $A$ are the enthalpy change due to combustion, the specific heat at constant pressure and the pre-exponential factor, respectively. This parameter, important to the current study, specifies the ratio of the rate of reaction to the rate of fluid convection.

The system of equations (2.3), together with the continuity and momentum equations, are solved using nearly the same numerical scheme as in Nichols et al. (2007). Briefly, sixth-order compact schemes are used to compute spatial derivatives on a non-uniform mesh in cylindrical coordinates, and timestepping is implemented using a fourth-order Runge-Kutta scheme. A projection method is used to ensure that the constraint imposed by the continuity equation is satisfied at every timestep. A computational domain with axial extent $0<x<10$ and radial extent $-5<r<5$ is considered, where $x$ and $r$ are non-dimensionalized with respect to the jet diameter $d$. To adequately resolve the flames, this domain is discretized by $1024 \times 512$ grid points in the $x$ - and $r$-directions, respectively. A non-dimensional timestep of $\Delta t=0.0025$ is used so that a typical simulation with $0<t<500$ requires 200000 timesteps.

\section{Simulation results}

Results of the five different reacting simulations listed in table 1 in are shown in figure 2. Each image represents an instantaneous snapshot of the reaction rate field taken at non-dimensional time $t=500$, long after any transient effects have died away. To show all parts of the flame, colour contours of $\ln (\dot{w})$ are displayed. In all of the flames, triple flames are observed to form at the lifted flame base, consisting of a short lean branch, a long rich branch, and a diffusion flame which forms around the stoichiometric surface shown in black. From top to bottom, $D a$ is incrementally increased. As $D a$ increases, the liftoff height $H$ decreases, as expected. For $D a=3,4,5 \times 10^{5}$, corresponding to the top three images, $H$ is sufficiently great that the flame remains unstable, owing to the pocket of absolute instability of the non-reacting premixing zone just upstream of the flame. For $D a=6,7 \times 10^{5}$, however, $H$ is sufficiently small that the flame appears stable.

The nature of the lifted flame instability can also be seen in figure $3(a)$ which shows time histories of the instantaneous liftoff height. The blue curve, corresponding to $D a=3 \times 10^{5}$, shows that the flame oscillates about a mean liftoff height of $H_{m}=1.231$ with a non-dimensionalized frequency of $S t=0.238$. From the top spectrum shown in figure $3(b)$, we observe that only this frequency and its harmonics are contained within the signal. As $D a$ is decreased, the red and green curves corresponding to $D a=4,5 \times 10^{5}$, respectively, show a decrease in liftoff height. The frequency of the 


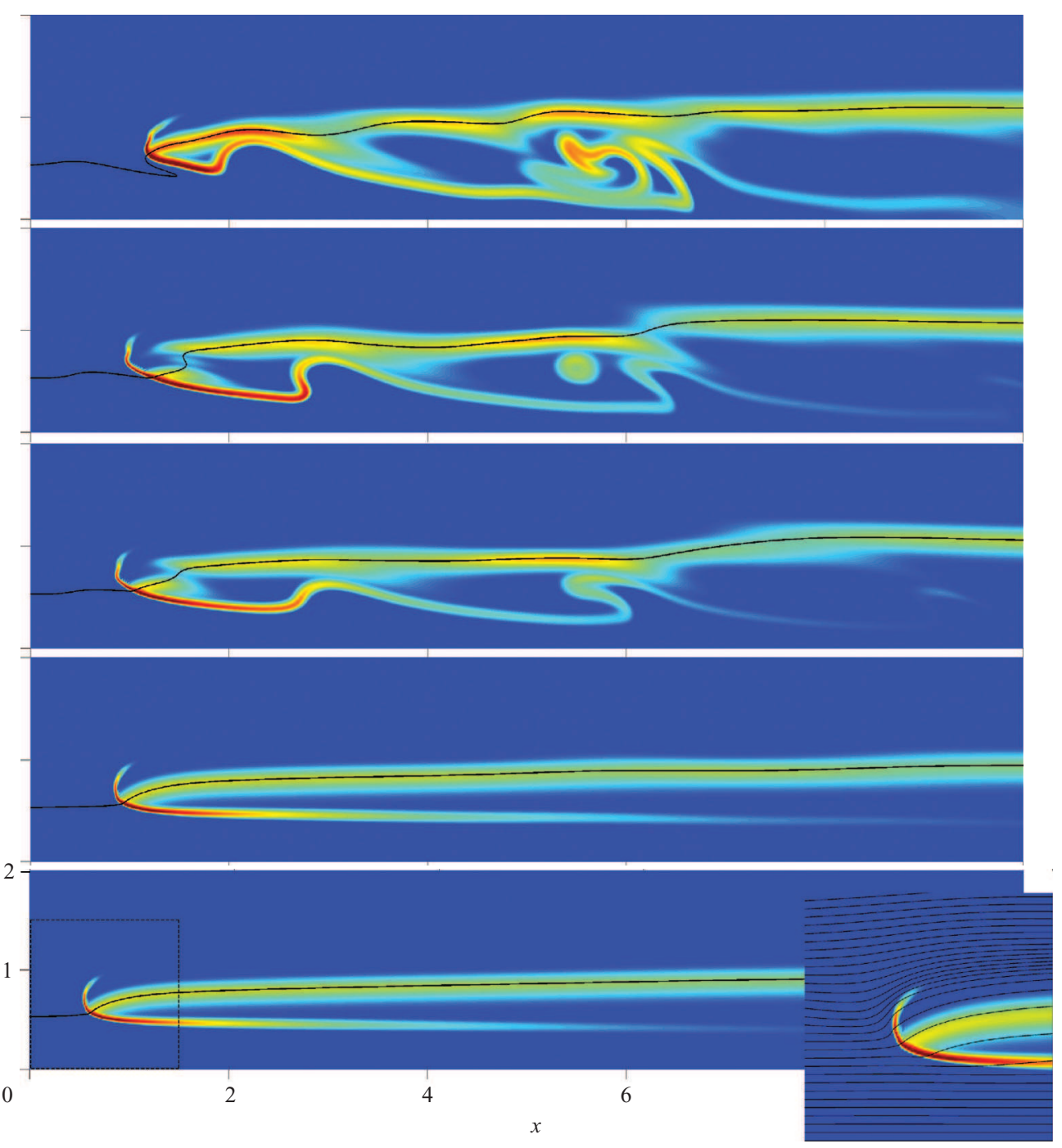

FigURE 2. Instantaneous snapshots of lifted flames from five different simulations at time $t=500$. Top to bottom, $D a=3.0,4.0,5.0,6.0,7.0 \times 10^{5}$. Colour contours of $\ln (\dot{w})$ are displayed showing the interaction of the triple flame with the stoichiometric surface (black line). The inset is an enlargement of the region bounded by the dashed lines, together with fluid streamlines.

instability is observed to increase with decreasing liftoff height. In these cases, a lowfrequency fluctuation is observed to appear at $S t=0.029,0.025$ for the $D a=4,5 \times 10^{5}$ cases, respectively. Lastly, the spectrum of the $D a=6 \times 10^{5}$ flame is displayed in figure $3(b)$, showing that it still has the same shape as the spectra observed for $D a=4,5 \times 10^{5}$, although its amplitude is greatly reduced (so the oscillations are barely visible in $3(a))$. We therefore consider this case to be marginally unstable. The spectrum for $D a=7 \times 10^{5}$ is not shown because all of the spectral power is in the lowest frequency component since this case is stable.

To understand the physical mechanism of the lifted flame base stabilization in the unstable case $D a=4 \times 10^{5}$, we consider in figure 4 a time sequence of the flame base taken at equally spaced phase intervals over one cycle of the oscillation. 


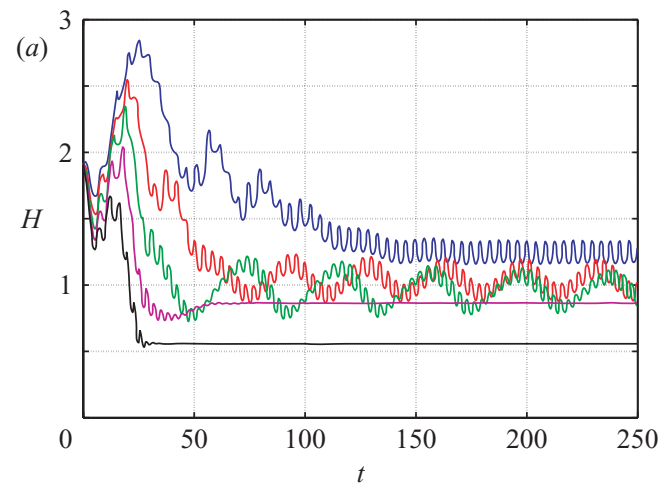

(b)
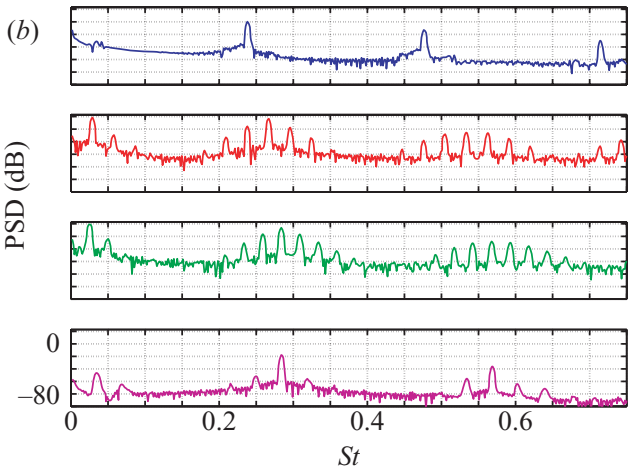

FIGURE 3. (a) Time histories of the liftoff height for the five reacting simulations with $D a=3,4,5,6,7 \times 10^{5}$ represented by blue, red, green, magenta, and black, respectively. $(b)$ Power spectral density vs. Strouhal number for four of the signals displayed in $(a)$. As $D a$ increases, the liftoff height decreases, stabilizing the flow as the flame restricts the pocket of absolute instability.
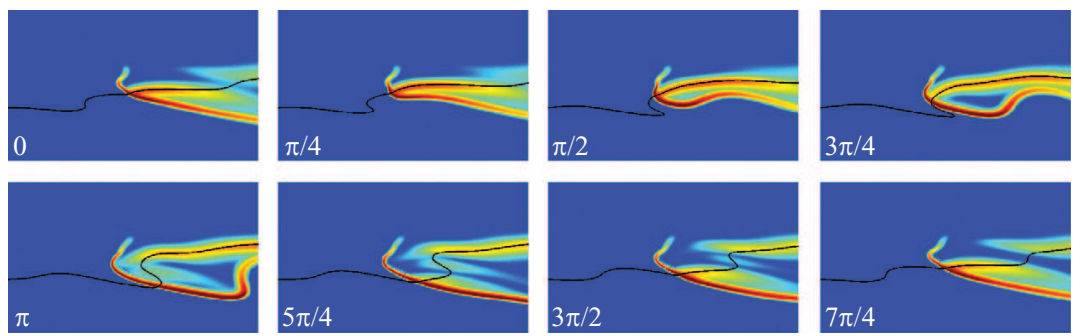

FIgURE 4. A time sequence of the unsteady flame base for $D a=4 \times 10^{5}$. The images show the same as figure 2 , taken at equal phase intervals of $\pi / 4$.

In the first image, a triple flame is stabilized about the oncoming perpendicular stoichiometric surface shown by the black line. At the same time, the stoichiometric surface is observed to start to fold upstream of the flame, owing to the beginning of a Kelvin-Helmholtz vortex rollup. As time progresses, this fold grows and convects downstream until it meets the flame base as shown in the image at $3 \pi / 4$. At this point, the stoichiometric line is no longer perpendicular to the flame base, and the flame is able to propagate as a premixed flame, through the vortex core. After the vortex, represented by the folding of the stoichiometric surface, passes through the flame, the triple flame again forms perpendicular to the stoichiometric surface, and the cycle repeats.

The premixed flame propagation in the above cycle allows the flame to move much closer to the nozzle than otherwise possible. For comparison, we have computed steady, but unstable, solutions to the reacting low-Mach-number equations by applying the technique of selective frequency damping (SFD) (Åkervik et al. 2006) to the unstable flames. The SFD method effectively applies a low-pass filter to the simulations so that the global oscillations are damped and the solution is driven to a steady state. Figure 5 shows a comparison between the liftoff height $H_{s}$ obtained from a steady SFD solution and the mean liftoff height $H_{m}$ obtained from time averaging 


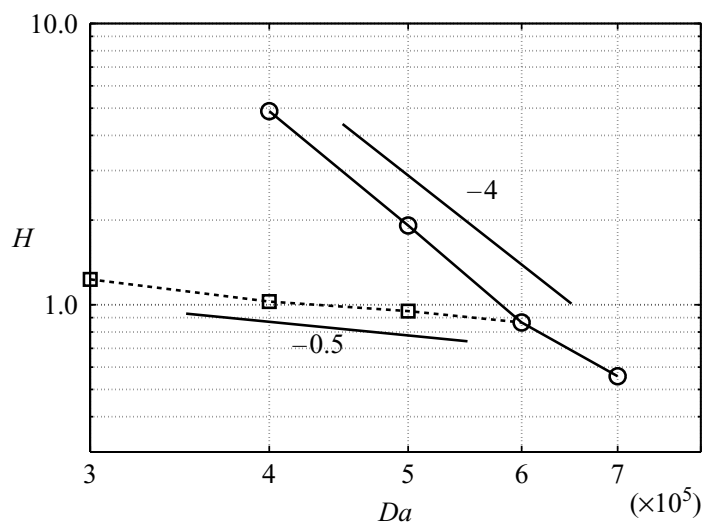

FIGURE 5. A log-log plot of liftoff height from steady SFD solutions (circles) and time-averaged liftoff height (squares) vs. $D a$. As $D a$ is decreased below $6 \times 10^{5}$, the flame becomes globally unstable, so the two curves diverge. For $D a \geqslant 6 \times 10^{5}$ the two liftoff heights coincide.

the unstable solutions. The curves diverge for $D a<6 \times 10^{5}$, which represents the regime of instability. In this regime, it appears from the log-log plot that $H_{s} \sim D a^{-4}$ while $H_{m} \sim D a^{-0.5}$, so their difference rapidly becomes large with decreasing $D a$ : for $D a=4 \times 10^{5}, H_{s}$ is five times $H_{m}$. The observation that $H_{m} \sim D a^{-0.5}$ in the unstable flames provides further evidence of the premixed flame propagation in part of the above cycle since the premixed laminar flame speed also scales as $s_{L} \sim D a^{-0.5}$.

\section{Local stability analysis}

To identify the pocket of absolute instability within the premixing zone, we analyse the local stability of the steady solutions obtained for $D a=0,5,6,7 \times 10^{5}$. For $D a=7 \times 10^{5}$ the flame is globally stable, so a steady solution is obtained from direct simulation. In the other three cases, the flow is globally unstable, and for these we apply SFD to obtain steady (but unstable) solutions about which to linearize. It is also possible to linearize about mean profiles, but we have chosen to use the SFD profiles for two reasons. First, we find numerically that the no-flame SFD solution $(D a=0)$ is a better match to the premixing zone upstream of a steady flame than the mean no-flame solution to a time-averaged flame. Quantitatively, $\left\|Z_{s, n}-Z_{s, r}\right\|_{\infty}<0.01$ over the first $80 \%$ of the premixing zone of the $D a=5 \times 10^{5}$ SFD flame, whereas $\left\|Z_{m, n}-Z_{m, r}\right\|_{\infty}<0.01$ for only the first $30 \%$ of the premixing zone of the corresponding time-averaged flame. Here, the subscripts $s, m, n, r$ denote SFD, mean, non-reacting and reacting, respectively. Second, since the SFD solutions are solutions to the exact reacting low-Mach-number equations, they preserve thinflame physics whereas the mean flow solutions lead to unphysical thick 'flames'.

From the steady solutions, we extract base profiles $u_{x}(r), Z(r)$ and $T(r)$ at each axial location $x$, and invoke the assumption of locally parallel flow. The complex spatial eigenvalue spectrum of each resulting linearized system is obtained through a matrix method using a spectral discretization (Schmid \& Henningson 2001; Nichols 2005). Absolute versus convective instability is determined by identifying a pinch point $\left(\omega_{0}, \alpha_{0}\right)$ of the complex dispersion relation. The frequency of the absolute mode is given by $\omega_{0, r}=\operatorname{Re}\left\{\omega_{0}\right\}$, and the absolute growth rate is given by $\omega_{0, i}=\operatorname{Im}\left\{\omega_{0}\right\}$. If $\omega_{0, i}>0$ the flow is said to be absolutely unstable whereas if $\omega_{0, i}<0$ the flow is convectively unstable. Since the absolute mode of a round variable-density jet is axisymmetric (Monkewitz \& Sohn 1988), we consider only axisymmetric perturbations here. 

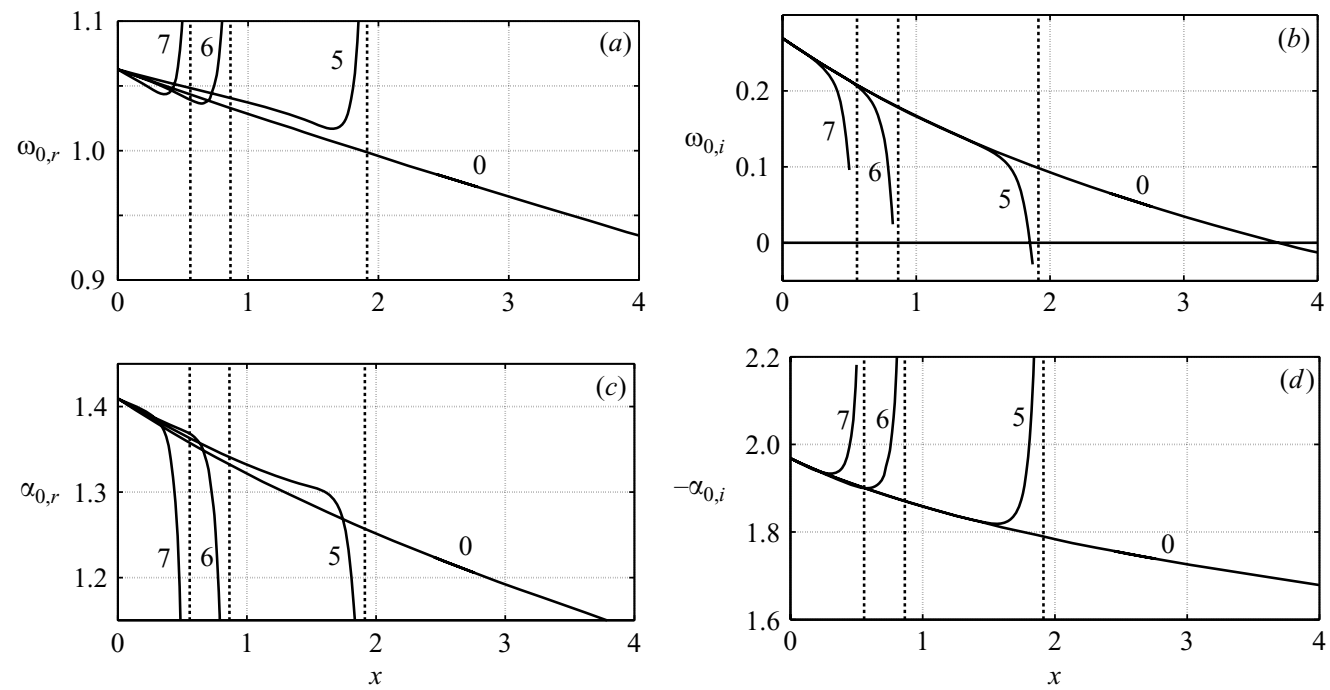

FIGURE 6. Axial development of the $(a)$ frequency $\omega_{0, r},(b)$ growth rate $\omega_{0, i},(c)$ wavenumber $\alpha_{0, r}$ and $(d)$ spatial growth rate $-\alpha_{0, i}$ of the absolute mode. The curves labelled $0,5,6$ and 7 correspond to the four simulations considered, with $D a=0,5,6,7 \times 10^{5}$, respectively.

Figure 6 shows the axial development of $\omega_{0}$ and $\alpha_{0}$ for four different simulation configurations $\left(D a=0,5,6,7 \times 10^{5}\right)$. In all cases, the flow profiles used at the nozzle begin as absolutely unstable with $\omega_{0, r}=1.063$ corresponding to $S t_{0}=0.169$. The fact that the frequency of the global mode of the non-reacting jet (table 1 ) is $25 \%$ greater than the freqency of the absolute mode agrees well with the results of Lesshafft et al. (2006) who found a $20 \%$ difference at the same density ratio, although for slightly thicker shear layers. The axial development of $\omega_{0}$ and $\alpha_{0}$ is found by applying a continuation algorithm to the stability solver over the continuously varying base profiles in $x$. At the end of each continuation, we verify that $\alpha_{0}$ remains the dominant $\alpha^{+} / \alpha^{-}$pinch point by considering a map (not shown) of $\omega_{i}$ contours in the complex $\alpha$-plane (see Juniper \& Candel 2003). In the non-reacting case, the absolute growth rate $\omega_{0, i}$ decreases gradually and eventually passes below 0 at $X=3.71$, which marks the trailing edge of the pocket of absolute instability, where the non-reacting jet transitions from absolute to convective instability.

In contrast to the non-reacting case, the local stability properties of the reacting jets undergo a sharp transition when the flame base is encountered. In figure 6 , the steady liftoff heights $H_{s}$ for the three reacting cases are represented by the dashed vertical lines. Just upstream of the flame, we observe a sudden increase in the absolute frequency $\omega_{0, r}$, a sudden decrease in the absolute growth rate $\omega_{0, i}$, and a sudden decrease in the wavenumber $\alpha_{0, r}$ of the absolute mode. These observations can be understood as effects of an axial acceleration of the shear layer through the flame surface, owing to an expansion from heat release. Boulanger et al. (2003) found that the fluid begins to deflect (see figure 2, inset) and accelerate a short distance ahead of the lifted flame base, and that this expansion effect is an important consideration in predicting the liftoff height. In this paper, we point out that expansion also strongly influences the local stability properties of the flow, even ahead of the flame base. First, acceleration due to expansion yields shorter time scales and longer length scales which produce higher frequencies and lower wavenumbers, as observed. Expansion also diffuses vorticity which tends to stabilize the flow, explaining the decrease in $\omega_{0, i}$. 
The increase of the spatial growth rate $-\alpha_{0, i}$ in this zone can be understood as a combination of the previous three effects through the complex dispersion relation.

The sharp decrease in $\omega_{0, i}$ represents an abrupt transition in the local properties from absolute to convective instability. Because this transition is sharp, we interpret the acceleration through the flame base at $x=H$ to act as an internal Neumann boundary condition to the nearly parallel flow upstream. For finite-sized systems governed by the Ginzburg-Landau equation (which serves as a good model for absolutely unstable flows, see Huerre \& Monkewitz 1990), Chomaz \& Couairon (1999) have shown that such an axial bounding has the potential to stabilize an otherwise absolutely unstable flow. Note that this internal boundary does not preclude waves from being transmitted through $x=H$ to the flame downstream. Since the flame with $D a=7 \times 10^{5}$ is stable, we conclude that the flame downstream of $x=H$ does not support intrinsic self-sustaining oscillations. Therefore, flame oscillations in the unstable cases must be driven by self-sustaining oscillations in the absolutely unstable premixing zone $x<H$. In summary, the premixing zone serves as a wavemaker, from which waves propagate downstream causing flame flicker. For low $H$, truncation effects stabilize the wavemaker, and thus the entire flow.

\section{Conclusions}

By direct numerical simulation, we have confirmed our hypothesis that a globally unstable variable-density fuel jet can be stabilized by a lifted flame with a sufficiently low liftoff height. The case above with $D a=7 \times 10^{5}$ serves as an example of a flow containing a pocket of absolute instability but which is globally stable. This demonstrates that while the existence of a region of absolute instability may be a necessary condition for global instability, it is not a sufficient condition (see also Pier \& Huerre 2001).

For larger liftoff heights, however, the flow remains globally unstable owing to the unfettered pocket of absolute instability. Although lifted flame oscillations have been linked to effects of buoyancy in the case of propane (heavy) jets (Won et al. 2002; Chung 2007), the current results suggest that for light jets, the wavemaking region in the premixing zone provides another mechanism for producing flame flicker, even in the absence of gravity. In this case, we observe the instability of a jet which imparts its frequency to the flame downstream, rather than an intrinsic flame instability. For example, this interpretation may explain the oscillations observed in numerical simulations of a lifted flame on a round methane jet discussed by Kaplan, Oran \& Baek (1994).

A secondary conclusion of this paper is that the presence of instability significantly influences liftoff height. Using the SFD technique, we compared the liftoff height $H_{s}$ of a steady, but unstable, flame to the mean liftoff height $H_{m}$ of an oscillating flame at the same Damköhler number. For $D a=4 \times 10^{5}$, we found a factor of five difference between $H_{s}$ and $H_{m}$. By considering a time sequence of the lifted flame base, we observed that when a Kelvin-Helmholtz vortex arrived at the flame base, the flame propagated in a premixed mode through the vortex core. This allowed higher average flame speeds than those given by edge-flame theory, producing lower liftoff heights than in a steady flame.

The authors are grateful to Professors J.-M. Chomaz and M. Juniper for fruitful discussions throughout the course of this work and to the reviewers for helpful suggestions. This research has been supported by the ANR Chaires d'Excellence program. 


\section{REFERENCES}

Åkervik, E., Brandt, L., Henningson, D. S., Heppffner, J., Marxen, O. \& Schlatter, P. 2006 Steady solutions of the Navier-Stokes equations by selective frequency damping. Phys. Fluids 18, 068102.

Boulanger, J., Vervisch, L., Reveillon, J. \& Ghosal, S. 2003 Effects of heat release in laminar diffusion flames lifted on round jets. Combust. Flame 134, 355-368.

Chomaz, J.-M. 2005 Global instabilities in spatially developing flows: non-normality and nonlinearity. Annu. Rev. Fluid Mech. 37, 357-392.

Chomaz, J.-M. \& Couairon, A. 1999 Against the wind. Phys. Fluids 11, 2977-2983.

Chung, S. H. 2007 Stabilization, propagation and instability of tribrachial triple flames. Proc. Combust. Inst. 31, 877-892.

Ghosal, S. \& Vervisch, L. 2000 Theoretical and numerical study of a symmetrical triple flame using the parabolic flame path approximation. J. Fluid Mech. 415, 227-260.

Ghosal, S. \& Vervisch, L. 2001 Stability diagram for lift-off and blowout of a round jet laminar diffusion flame. Combust. Flame 123, 646-655.

Huerre, P. \& Monkewitz, P. A. 1990 Local and global instabilities in spatially developing flows. Annu. Rev. Fluid Mech. 22, 473.

Joedicke, A., Peters, N. \& Mansour, M. 2005 The stabilization mechanism and structure of turbulent hydrocarbon lifted flames. Proc. Combust. Inst. 30, 901-909.

Juniper, M. P. \& CANDEL, S. M. 2003 The stability of ducted compound flows and consequences for the geometry of coaxial injectors. J. Fluid Mech. 482, 257-269.

Kaplan, C. R., Oran, E. S. \& BaEK, S. W. 1994 Stabilization mechanism of lifted jet diffusion flames. In Twenty-Fifth Symp. Intl on Combustion, pp. 1183-1189. The Combustion Institute, Pittsburgh.

Lesshafft, L., Huerre, P., Sagaut, P. \& Terracol, M. 2006 Nonlinear global modes in hot jets. J. Fluid Mech. 554, 393-409.

Linán, A., Fernández-Tarrazo, E., Vera, M. \& Sánchez, A. L. 2005 Lifted laminar jet diffusion flames. Combust. Sci. Technol. 177, 933-953.

McMurtry, P. A. 1987 Direct numerical simulations of a reacting mixing layer with chemical heat release. $\mathrm{PhD}$ thesis, University of Washington.

Michalke, A. 1984 Survey on jet instability theory. Prog. Aerospace Sci. 21, 159-199.

Monkewitz, P. A. \& Sohn, K. D. 1988 Absolute instability in hot jets. AIAA J. 26, 911-916.

Nichols, J. W. 2005 Simulation and stability analysis of jet diffusion flames. PhD thesis, University of Washington.

Nichols, J. W., Schmid, P. J. \& Riley, J. J. 2007 Self-sustained oscillations in variable-density round jets. J. Fluid Mech. 582, 341-376.

Peters, N. \& Williams, F. A. 1983 Liftoff characteristics of turbulent jet diffusion flames. AIAA J. 21, 423-429.

Pier, B. \& Huerre, P. 2001 Nonlinear synchronization in open flows. J. Fluids Structures 15, 471-480.

Poinsot, T. \& Veynante, D. 2005 Theoretical and Numerical Combustion, 2nd edn. Flourtown, PA: Edwards.

Schmid, P. J. \& Henningson, D. S. 2001 Stability and Transition in Shear Flows. Springer.

Sripakagorn, P., Mitarai, S., Kosály, G. \& Pitsch, H. 2004 Extinction and reignition in a diffusion flame: a direct numerical simulation study. J. Fluid Mech. 518, 231-259.

Tap, F. A. \& VeYnante, D. 2005 Simulation of flame lift-off on a diesel jet using a generalized flame surface density modeling approach. Proc. Combust. Inst. 30, 919-926.

VANQUiCKenborne, L. \& VAN Tiggelen, A. 1966 Stabilization mechanism of lifted diffusion flames. Combust. Flame 10, 59-69.

Won, S. H., Kim, J., Hong, K. J., ChA, M. S. \& Chung, S. H. 2005 Stabilization mechanism of lifted flame edge in the near field of coflow jets for diluted methane. Proc. Combust. Inst. 30, 339-347.

Won, S. H., Kim, J., Shin, M. K., Chung, S. H., Fujita, O., Mori, T., Choi, J. H. \& Ito, K. 2002 Normal and microgravity experiment of oscillating lifted flames in coflow. Proc. Combust. Inst. 29, 37-44. 